\title{
Lie symmetries for systems of evolution equations
}

\author{
Andronikos Paliathanasis ${ }^{1,2 *}$ \\ ${ }^{1}$ Instituto de Ciencias Físicas y Matemáticas, \\ Universidad Austral de Chile, Valdivia, Chile \\ ${ }^{2}$ Institute of Systems Science, Durban University of Technology \\ Durban 4000, Republic of South Africa \\ Michael Tsamparlis ${ }^{3 \dagger}$ \\ ${ }^{3}$ Faculty of Physics, Department of Astronomy-Astrophysics-Mechanics, \\ University of Athens, Panepistemiopolis, Athens 157 83, Greece
}

September 25, 2018

\begin{abstract}
The Lie symmetries for a class of systems of evolution equations are studied. The evolution equations are defined in a bimetric space with two Riemannian metrics corresponding to the space of the independent and dependent variables of the differential equations. The exact relation of the Lie symmetries with the collineations of the bimetric space is determined.
\end{abstract}

Keywords: Lie symmetries; Evolution equation; Collineations

\section{Introduction}

Lie symmetries is a powerful method for the determination of solutions in the theory of differential equations. A Lie symmetry is important as it provides invariants which can be used to write a new differential equation with less degree of freedom. Furthermore, a solution of such an equation is also a solution of the original differential equation [1,2]. The reduction process is the main application of Lie symmetries, however, it is not a univocal approach. Symmetries can be used for the determination of conservation currents [3], for the classification of differential equations [4 10] and for the reconnaissance of some well-known systems [11 15].

In the recent literature, it has been shown that there is a close relation between the Lie symmetries of a second order differential equation and the geometry of the space where motion occurs. For example, the conservation of energy and angular momentum in Newtonian Physics is a result of the Lie point symmetries, generated by the Killing vectors of translations and rotations respectively. The general result for a holonomic autonomous dynamical system moving in a Riemannian space is that the Lie point symmetries of the equations of motion

\footnotetext{
*Email: anpaliat@phys.uoa.gr

$\dagger$ Email: mtsampa@phys.uoa.gr
} 
are generated by the special projective algebra of the kinematic metric, that is, the metric defined by the kinetic energy. [16 22]. Similar results have been derived for second-order partial differential equations [23-25].

The importance of the relation of Lie symmetries with the symmetry groups lies in that one can determine those symmetries, hence, reduce the problem of finding solutions for second order time independent differential equations by applying direct theorems of differential geometry [26 28]. Moreover, it has been shown [29] that the application of the geometric approach in constrained dynamical systems can be used to extract information during the quantization process 130 [33].

The present work is a natural extension of the approach initiated in [23,24, that is, we consider the problem of determining the Lie (point) symmetries for a class of systems of quasilinear evolution equations which define a bimetric manifold with two independent connections. The system of differential equations is assumed to be of the form

$$
Q^{A}\left(t, x^{k}, u^{C}, u_{, i}^{C}, u_{, i j}^{C}, u_{t}^{C}\right)=0
$$

in which

$$
Q^{A} \equiv g^{i j} u_{, i j}^{A}+g^{i j} \tilde{\Gamma}_{B C}^{A} u_{, i}^{B} u_{, j}^{C}-\Gamma^{i} u_{, i}^{A}+F^{A}\left(t, x^{k}, u^{C}\right)-u_{, t}^{A},
$$

where $u^{A}$ denotes the $m$-dependent variables, $\operatorname{dim}\left(u^{A}\right)=m, x^{k}$ are the $n$-independent variables, $\operatorname{dim}\left(x^{k}\right)=$ $n$, while $\Gamma^{i}=g^{j k} \Gamma_{j k}^{i}\left(x^{r}\right)$ and $\tilde{\Gamma}_{B C}^{A}=\tilde{\Gamma}_{B C}^{A}\left(u^{D}\right)$ are the two affine connections on the bimetric theory $\left\{g_{i j}, H_{A B}\right\}$. Metric $g_{i j}$ is defined in the space of the independent variables while $H_{A B}\left(u^{C}\right)$ defines the geometry of the space of dependent variables $u^{A}$. Moreover, we assume that the two spaces are minimally coupled, that is, $\left\{g_{i j}, H_{A B}\right\}=\left\{g_{i j}\left(x^{k}\right), H_{A B}\left(u^{C}\right)\right\}$. Finally, the function $F^{A}\left(t, x^{k}, u^{C}\right)$ counts for the interaction term of the metrics $g_{i j}, H_{A B}$ and $t$ denotes the "time" in which the system evolves.

Static solutions of the system (11) have been studied in 24. It was found that the Lie point symmetries of (11) are constructed by the conformal Killing vectors and the affine collineations of the metrics $g_{i j}$ and $H_{A B}$ respectively. The present letter is organized as follows.

In Section 2 we present the main results. We solve the symmetry conditions for the system (11) and show that the Lie point symmetries are generated by the elements of the homothetic algebra and the affine algebra of the two metrics which are defined by the differential equations. Three corollaries are presented for some cases of special interest. Finally, our discussion is given in Section 3.

\section{Lie point symmetries}

The lhs of the differential equation (11) can be seen as a vector field $Q^{A}$ in the space of variables $A=$ $A\left(t, x^{k}, u^{C}, u_{, i}^{C}, u_{, i j}^{C}, u_{t}^{C}\right)$. The condition is that the function $Q^{A}$ is invariant under the action of the one parameter point transformation

$$
\begin{aligned}
\bar{t} & =t+\varepsilon \xi^{t}\left(t, x^{k}, u^{B}\right) \\
\bar{x}^{i} & =x^{i}+\varepsilon \xi^{i}\left(t, x^{k}, u^{B}\right), \\
\bar{u}^{A} & =\bar{u}^{A}+\varepsilon \eta^{A}\left(t, x^{k}, u^{B}\right),
\end{aligned}
$$

is

$$
\mathbf{X}^{[2]} Q^{A}=0
$$

\footnotetext{
${ }^{1}$ For some interesting results on the quantization from symmetries see [34 [36] and references therein.
} 
where $\mathbf{X}^{[2]}$ is the second extension for the generator $X=\xi^{t} \partial_{t}+\xi^{i} \partial_{i}+\eta^{A} \partial_{A}$ in the space of both independent and dependent variables. In particular, the second extension is given by the formula [1,2]

$$
\mathbf{X}^{[2]}=\mathbf{X}+\eta_{\alpha}^{A} \partial_{u_{\alpha}^{A}}+\eta_{\alpha \beta}^{A} \partial_{u_{\alpha \beta}^{A}}
$$

where $\eta_{\alpha}^{A}$ and $\eta_{\alpha \beta}^{A}$ are defined as follows

$$
\begin{gathered}
\eta_{\alpha}^{A}=\eta_{, \alpha}^{A}+u_{, \alpha}^{B} \eta_{, B}^{A}-\xi_{, \alpha}^{\beta} u_{, \beta}^{A}-u_{, \beta}^{A} u_{, \alpha}^{B} \xi_{, B}^{\beta} \\
\eta_{\alpha \beta}^{A}=\eta_{, \alpha \beta}^{A}+2 \eta_{, B(\alpha}^{A} u_{, \beta)}^{B}-\xi_{, \alpha \beta}^{\gamma} u_{, \gamma}^{A}+\eta_{, B C}^{A} u_{, \alpha}^{B} u_{, \beta}^{C}-2 \xi_{,(\alpha|B|}^{\gamma} u_{\beta)}^{B} u_{, \gamma}^{A} \\
-\xi_{, B C}^{\gamma} u_{, \alpha}^{B} u_{, \beta}^{C} u_{, \gamma}^{A}+\eta_{, B}^{A} u_{, \alpha \beta}^{B}-2 \xi_{,(\beta}^{\gamma} u_{, \alpha) \gamma}^{A}-\xi_{, B}^{\gamma}\left(u_{, \gamma}^{A} u_{, \alpha \beta}^{B}+2 u_{, \beta}^{B} u_{, \alpha) \gamma}^{A}\right) .
\end{gathered}
$$

Considering condition, (6) , as a polynomial in the variables $\left(u^{A}\right)^{0},\left(u_{, \alpha}^{A}\right),\left(u_{, \alpha}^{A} u_{, \beta}^{B}\right),\left(u_{, \alpha \beta}^{A}\right)$ and $\left(u_{, \alpha \beta}^{A} u_{, \gamma}^{B}\right)$ we find the following symmetry conditions

$$
\begin{gathered}
\xi_{, i}^{t}=0, \xi_{, A}^{t}=0, \xi_{, A}^{i}=0 \\
F_{, k}^{A} \xi^{k}+F_{, t}^{A} \xi^{t}+F_{, B}^{A} \eta^{B}+\left(g^{i j} \eta_{; i j}^{A}-\eta_{, t}^{A}\right)=\lambda F^{A} \\
\left(-g^{k j} \xi_{, k j}^{i}-\Gamma_{, k}^{i} \xi^{k}+\xi_{, j}^{i} \Gamma^{j}+\lambda \Gamma^{i}+\xi_{, t}^{i}\right) \delta_{B}^{A}-\Gamma^{i} \eta_{, B}^{A}+2 g^{i j}\left(\eta_{\mid B}^{A}\right)_{, j}=0 \\
\left(L_{\xi} g^{i j}-\lambda g^{i j}\right) \tilde{\Gamma}_{B C}^{A}+\tilde{\Gamma}_{B C, D}^{A} \eta^{D}+g^{i j} \eta_{, B C}^{A}+2 g^{i j} \tilde{\Gamma}_{K C}^{A} \eta_{, C}^{K}=0 \\
\delta_{B}^{A} L_{\xi} g^{i j}=\left(\lambda-\eta_{, B}^{A}\right) g^{i j} \delta_{B}^{A} \\
\eta_{, B}^{A}=\left(\xi_{, t}^{t}-\lambda\right) \delta_{B}^{A}
\end{gathered}
$$

where "|" and ";" denote covariant derivative with respect to the connection $\tilde{\Gamma}_{B C}^{A}$ or $\Gamma_{j k}^{i}$ respectively, while $\mathcal{L}_{X}$ denotes Lie derivative. The symmetry conditions are similar to those derived in [24, therefore, we follow the same procedure in their solution.

Condition (14) becomes

$$
L_{\xi} g_{i j}=\left(\lambda-\eta_{, B}^{A}\right) g_{i j}
$$

which means that $\xi^{i}$ is a conformal Killing vector $(\mathrm{CKV})$ field for the metric $g_{i j}$ with conformal factor $\psi=$ $\frac{1}{2}\left(\lambda-\eta_{, B}^{A}\right)$. However, from (15) we have that $\psi=\psi(t)$ only such that $\xi^{t}=2 \psi \int T(t) d t$. Hence, $\xi^{i}=$ $T(t) \zeta^{i}$, where $\zeta^{i}$ is a homothetic vector $(\mathrm{HV})$ of $g_{i j}$.

From (13) we have that

$$
\mathcal{L}_{\eta} \tilde{\Gamma}_{B C}^{A}=0
$$

which implies that $\eta^{A}$ is an affine collineation (AC) of the metric $H_{A B}$. These two conditions, namely (16) and (17) provide the geometric answer to the relation between Lie point symmetries of (11) and collineations of the underlying space.

The remaining symmetry conditions produce constraints on these collineations which should be satisfied in order the latter to be Lie point symmetries for the system (11).

We rewrite condition (12) as follows

$$
\left(\eta_{\mid B}^{A}\right)_{, j}=-T_{, t} \zeta_{i} \delta_{B}^{A}
$$

\footnotetext{
${ }^{2}$ The Greek indices $a, \beta, \gamma$ count on all the independent parameters $x^{i}$ and $t$.
} 
from which follows that

$$
\eta_{\mid B}^{A}=-T_{, t} \bar{\zeta} \delta_{B}^{A}+Z\left(t, u^{C}\right)
$$

where $\bar{\zeta}_{, i}$ denotes a gradient HV of $g_{i j}$, otherwise $T(t)=$ const. such that $T_{, t}=0$. From (19) and (17) it follows that

$$
\eta^{A}=-T_{, t} \bar{\zeta} Y^{A}\left(u^{C}\right)+Z\left(t, u^{C}\right)+\sigma^{A}\left(t, x^{k}\right)
$$

where $Z^{A}$ is an $\mathrm{AC}$ of $H_{A B}$ and $Y^{A}$ is a proper gradient $\mathrm{HV}$ of $H_{A B}$.

Finally, condition (11) becomes

$$
\mathcal{L}_{\xi^{i}} F^{A}+2 \psi \int T(t) d t F_{, t}^{A}+2 \psi T(t) F^{A}-T_{, t} \bar{\zeta} \mathcal{L}_{Y} F^{A}+\mathcal{L}_{Z} F^{A}+\left(g^{i j} \eta_{; i j}-\eta_{, t}\right)=0
$$

The last condition is the one which constrains the collineations of the bimetric manifold with the function $F^{A}$ in order for the collineations to generate a Lie point symmetry for the system (11). The following theorem is the main result of this work and a natural extension of [24] for systems of evolution equations.

Theorem 1 The Lie point symmetries for the system of evolution equations (1) are generated by the collineations of the bimetric $\left\{g_{i j}, H_{A B}\right\}$. In particular, the generic form for the Lie symmetry vector is given by the formula

$$
X=2 \psi \int T(t) d t \partial_{t}+T(t) \zeta^{i}\left(x^{k}\right) \partial_{i}+\left(Z^{A}\left(t, u^{A}\right)-T_{, t} \bar{\zeta} Y^{A}\left(u^{C}\right)+\sigma^{A}\left(t, x^{k}\right)\right) \partial_{A}
$$

where $\zeta^{i}$ is an element of the Homothetic algebra of $g_{i j}$ with homothetic factor $\psi, \bar{\zeta}$ is a gradient homothetic vector field of $g_{i j}, Z^{A}$ and $Y^{A}$ are elements of the affine and homothetic algebra of the metric $H_{A B}$ respectively. The collineations and the functions $T(t), \sigma\left(t, x^{k}\right)$ are constrained by condition (21).

In the case where the bimetric does not admit any gradient $\mathrm{HV}$, it follows:

Corollary 2 The generic Lie point symmetry for the system of evolution equations (1) in which $g_{i j}$ and $H_{A B}$ do not admit a gradient $H V$ is

$$
X=2 \psi t \partial_{t}+\zeta^{i}\left(x^{k}\right) \partial_{i}+\left(Z^{A}\left(t, u^{A}\right)+\sigma^{A}\left(t, x^{k}\right)\right) \partial_{A},
$$

where $\zeta^{i}, Z^{A}$ and $\sigma\left(t, x^{k}\right)$ are constrained with the condition

$$
\mathcal{L}_{\xi^{i}} F^{A}+2 \psi t F_{, t}^{A}+2 \psi F^{A}+\mathcal{L}_{Z} F^{A}+\left(g^{i j} \sigma_{; i j}^{A}-\sigma_{, t}^{A}\right)-Z_{, t}^{A}=0 .
$$

In case where there is no interaction term $F^{A}$ in the system (10), that is $F^{A}\left(t, x^{k}, u^{C}\right)=0$, condition (21) becomes

$$
\left(g^{i j} \sigma_{; i i}^{A}-\sigma_{, t}^{A}\right)+\left(\psi T_{, t}-T_{, t t} \bar{\zeta}\right) Y^{A}-Z_{, t}^{A}=0
$$

that is

$$
\psi T_{, t} Y^{A}-Z_{, t}^{A}=0, T_{, t t} \bar{\zeta} Y^{A}=0,
$$

and

$$
g^{i j} \sigma_{; i i}^{A}-\sigma_{, t}^{A}=0 .
$$

From (26) it follows that $T_{, t t}=0$, that is $T(t)=T_{0}+T_{1} t$, while from (26) we have that $Z^{A}=\psi T_{1} t Y^{A}$ or $Z^{A} \neq Y^{A}$ and $T_{1}$. Condition (27) provides an infinite number of trivial symmetries, which is a well-known result for the heat equation. Thus, from Theorem 1 follows: 
Corollary 3 The generic Lie point symmetry for the system of free evolution equations

$$
g^{i j} u_{, i j}^{A}+g^{i j} \tilde{\Gamma}_{B C}^{A} u_{, i}^{B} u_{, j}^{C}-\Gamma^{i} u_{, i}^{A}+F^{A}\left(t, x^{k}, u^{C}\right)-u_{, t}^{A}
$$

is generated by the $H V$ of the metric $g_{i j}$ and the ACs of the connection $\tilde{\Gamma}_{B C}^{A}$ and it is given by the following formula

$$
\begin{gathered}
X=\left(\bar{\psi}\left(T_{1} t^{2}+T_{0} t\right)+\alpha_{1} \psi t+\alpha_{0}\right) \partial_{t}+\left(\bar{\psi}\left(T_{1} t+T_{0}\right) \bar{\zeta}^{, i}\left(x^{k}\right)+\alpha_{1} \zeta^{i}\right) \partial_{i}+ \\
+\left(Z^{A}\left(t, u^{A}\right)-\bar{\psi} T_{1}(t+1) \bar{\zeta} Y^{A}\left(u^{C}\right)+\sigma^{A}\left(t, x^{k}\right)\right) \partial_{A}
\end{gathered}
$$

in which $\zeta^{i}, \bar{\zeta}^{, i}$ are elements of the Homothetic algebra of $g_{i j}$ with Homothetic factors $\psi$ and $\bar{\psi}$, where $\bar{\zeta}^{i}$ is a gradient vector field. $Z^{A}$ is an affine collineation of $\tilde{\Gamma}_{B C}^{A}$ while $Y^{A}$ is a $H V$ for the metric $H_{A B}$. Finally, $\sigma^{A}\left(t, x^{k}\right)$ solves the system of independent linear evolution equations 27).

In the special scenario in which $\left\{g_{i j}, H_{A B}\right\}$ are metrics of maximally symmetric spaces with nonzero curvature from Corollaries 2 and 3 , we have:

Corollary 4 The generic Lie point symmetry for the system of free evolution equations of the form (28) where $\left\{g_{i j}, H_{A B}\right\}$ are metrics of maximally symmetric spaces with nonzero curvature is given as below

$$
X=\alpha_{0} \partial_{t}+\zeta^{i} \partial_{i}+\left(Z^{A}\left(t, u^{A}\right)+\sigma^{A}\left(t, x^{k}\right)\right) \partial_{A}
$$

where $\zeta^{i}$ and $Z^{A}$ are the $\frac{n(n+1)}{2}$ and $\frac{m(m+1)}{2}$ Killing vectors of the spaces $\left\{g_{i j}, H_{A B}\right\}$. Finally, $\sigma^{A}\left(t, x^{k}\right)$ solve the system of independent linear evolution equations (27).

For the proof of Corollary 4 we remind to the reader that maximally symmetric spaces of nonzero curvature do not admit a HV and ACs.

At this point, we omit the demonstration of the application of the derived results. The same approach which has been presented in [24] can be applied.

\section{Conclusion}

This work focuses on the relation between geometry and algebraic properties of differential equations. The system of evolution equations (11) is defined by a bimetric theory where the two Riemannian spaces define the spaces of the independent and dependent variables.

The main result of this work is Theorem 1 in which we construct the generic form of the Lie point symmetries for the system (11) and we transfer the problem of the determination of point Lie symmetries to a geometric problem. In particular, the geometric construction of the bimetric theory defines the existence, the number and the form of the Lie point symmetries.

Because the point transformation (3)-(5) has components in the spaces of the independent and the dependent variables, it follows that it is constructed by the HV and the ACs for the two metrics which define the bimetric theory. There are various differences with the quasilinear system we studied in 24 the main one being that in 24 the CKVs of the metric $g_{a b}$ generate symmetries of the system. That means that in the case of static solutions of the system (11) the new "Type-II" hidden symmetries [37,38] are generated by the proper CKVs of the metric $g_{i j}$, a result which generalizes the corresponding result of the one-dimensional case [39]. 
With the present work we complete our analysis on the relation between geometry and Lie point symmetries of differential equations of second-order of physical interest. There are various ways through which one could extend that geometric approach by studying other families of differential equations or studying higher-

order symmetries. A review on the geometric approach of the symmetries of differential equations where some extensions will be presented is in progress.

\section{Acknowledgements}

The research of AP was supported by FONDECYT postdoctoral grant no. 3160121. AP thanks the University of Athens for the hospitality provided while part of this work took place.

\section{References}

[1] H. Stephani, Differential Equations: Their Solutions Using Symmetry, Cambridge University Press, New York, (1989)

[2] G.W. Bluman and S. Kumei, Symmetries of Differential Equations, Springer-Verlag, New York, (1989)

[3] E. Noether, Nachr. v.d. Ges. d. Wiss. zu Gottingen 235, (1918)

[4] L. V. Ovsiannikov, Group analysis of differential equations, Academic Press, New York, (1982)

[5] N. Kallinikos and E. Meletidou, J. Phys. A: Math. Theor. 46, 305202 (2013)

[6] N. Kallinikos, Group classification of charged particle motion in stationary electromagnetic fields, arXiv:1707.05684

[7] K. Andriopoulos, P.G.L. Leach and G.P. Flessas, J. Math. Anal. Appl. 262, 256 (2001)

[8] A.H. Kara and F.M. Mahomed, Int. J. Theor. Phys. 34, 2267 (1995)

[9] S.-F. Shen and Y.-Y. Jin, Acta. Math. Appl. Sinica (English Series) 33, 345 (2017)

[10] S. Jamal, Gen. Relativ. Grav. 49, 88 (2017)

[11] P.G.L. Leach, J. Phys. A: Math. Gen. 13, 1991 (1980)

[12] S. Moyo and P.G.L. Leach,J. Math. Anal. Appl. 252, 840 (2000)

[13] W. Sarlet, F.M. Mahomed and P.G.L. Leach, J. Phys. A.: Math. Gen. 20, 277 (1987)

[14] F.M. Mahomed and P.G.L. Leach, Quaestiones Mathematicae 12, 121 (1989)

[15] M. Tsamparlis and A. Paliathanasis, J. Phys. A: Math. Theor. 45, 275202 (2012)

[16] G.H. Katzin and J. Levine, J. Math. Phys. 9, 8 (1968)

[17] G.H. Katzin and J. Levine, J. Math. Phys. 22, 1878 (1981)

[18] G.H. Katzin, J. Math. Phys. 14, 1213 (1973)

[19] A.V. Aminova, Sbornik Math. 186, 1711 (1995) 
[20] A.V. Aminova, Tensor N.S., 65, 62 (2000)

[21] M. Tsamparlis and A. Paliathanasis, Nonlinear Dyn. 62, 203 (2010)

[22] M. Tsamparlis and A. Paliathanasis, Gen. Relativ. Gravit. 42, 2957 (2010)

[23] A. Paliathanasis and M. Tsamparlis, J. Geom. Phys. 62, 2443 (2012)

[24] A. Paliathanasis and M. Tsamparlis, J. Geom. Phys. 107, 45 (2016)

[25] Y. Bozhkov and I.L. Freire, J. Diff. Eq. 249, 872 (2010)

[26] A. Paliathanasis and M. Tsamparlis, Phys. Rev. D. 90, 043529(2014)

[27] A. Paliathanasis, M. Tsamparlis and M.T. Mustafa, Int. J. Geom. Meth. Mod. Phys. 12, 1550033 (2015)

[28] A. Paliathanasis, M. Tsamparlis and M.T. Mustafa, Comm. Non. Sci. Num. Sim. 55, 68 (2018)

[29] T.Christodoulakis, N. Dimakis and P.A. Terzis, J. Phys. A: Math. Theor. 47, 095202 (2014)

[30] T. Christodoulakis, N. Dimakis, P.A. Terzis, Th. Grammenos, E. Melas and A. Spanou, J. Geom. Phys. 71, $127(2013)$

[31] T. Christodoulakis, N. Dimakis, P.A. Terzis and G. Doulis, Phys. Rev. D 90, 024052 (2014)

[32] N. Dimakis, A. Karagiorgos, T. Pailas, P.A. Terzis and T. Christodoulakis, Phys. Rev. D 95, 086016 (2017)

[33] A. Paliathanasis, M. Tsamparlis, S. Basilakos and J.D. Barrow, Phys. Rev. D 93, 043528 (2016)

[34] M.C. Nucci, Theor. Math. Phys. 168, 994 (2011)

[35] M.C. Nucci, J. Phys.: Conf. Ser. 482, 012032 (2014)

[36] M.C. Nucci and P.G.L. Leach, J. Non. Math. Phys. 17, 485 (2010)

[37] B. Abraham-Shrauner, K.S. Govinder and D.J. Arrigo, J. Phys. A: Math. Gen. 39, 5739 (2006)

[38] M.L. Gandarias, J. Math. Anal. Appl. 348, 752 (2008)

[39] M. Tsamparlis and A. Paliathanasis, J. Geom. Phys. 73, 209 (2013) 